TRANSACTIONS OF THE

AMERICAN MATHEMATICAL SOCIETY

Volume 226, 1977

\title{
ZERO-ONE LAWS AND THE MINIMUM OF A MARKOV PROCESS( ${ }^{(1)}$
}

BY

P. W. MILLAR

\begin{abstract}
If $\left\{X_{t}, t>0\right\}$ is a real strong Markov process whose paths assume a (last) minimum at some time $M$ strictly before the lifetime, then conditional on $I$, the value of this minimum, the process $\{X(M+t), t>0\}$ is shown to be Markov with stationary transitions which depend on $I$. For a wide class of Markov processes, including those obtained from Levy processes via time change and multiplicative functional, a zero-one law is shown to hold at $M$ in the sense that $\cap_{t>0}\{X(M+s), s<n=\sigma\{X(M)\}$, modulo null sets. When such a law holds, the evolution of $\{X(M+t), t>0\}$ depends on events before $M$ only through $X(M)$ and $I$.
\end{abstract}

1. Introduction. Let $X=\left\{X_{t}, t \geqslant 0\right\}$ be a real valued, right continuous strong Markov process. Let $R$ be a random time, such as the time point on the interval $[0, T]$ at which the paths $t \rightarrow X_{t}(\omega)$ achieve the minimum, or such as the last time before $T$ that the process leaves a given set. Let $\Re_{t}$ be the sigma field generated by the random variables $\{X(R+s), s \leqslant t\}$ and let $\Re_{0+}$ $=\cap_{t>0} \Re_{t}$. The main results of this paper give the precise conditions, for various $R$ and $X$, under which the sigma fields $\Re_{0+}$ and $\sigma\{X(R)\}$ are the same, modulo null sets. When such a phenomenon takes place, then conditional on $X(R)$, the sets in $\Re_{0+}$ have probability zero or one; for this reason, such results will be called zero-one laws at $R$. Most of the zero-one laws in this paper are established as a consequence of an even stronger assertion: if $\mathscr{R}_{s}^{\prime}$ is the sigma field generated by $\{X(R+u)-X(R), u<s\}$, and $\mathscr{R}_{0_{+}}^{\prime}=\cap_{s}>0 \Re_{s}^{\prime}$, then $\mathscr{R}_{0_{+}}^{\prime}$ is trivial.

When $R$ happens to be a stopping time, such zero-one laws were first proved by Hunt [8] for processes with stationary independent increments; Blumenthal [2] extended Hunt's result to more general Markov processes (under appropriate hypotheses) where it now goes under the name "Blumenthal zero-one law."

Received by the editors November 5, 1975.

AMS (MOS) subject classifications (1970). Primary 60J25, 60J30; Secondary 60F20, 60G17, 60G40, 60J40, $60 \mathrm{~J} 60$.

Key words and phrases. Zero-one laws, minimum, strong Markov process, stationary independent increments, random time, last exit time, path decomposition, $h$ path transform.

(1) This research was prepared with the support of National Science Foundation Grant No. MPS74-18967.

- American Mathematical Society 1977 
While the Blumenthal zero-one law holds quite generally, zero-one laws at, say, last exit times fail miserably a good deal of the time, even for otherwise well-behaved processes-see the eximples below. The need for zero-one laws at times $R$ different from stopping il. les first arose in [16] where the problem was to evaluate $\lim \inf _{t \downarrow 0}[X(R+t)-\quad(R)] / f(t)$ for a sure function $f$, where $R$ was a particular last exit time; if ${ }_{0} i_{0}$ were known so be trivial, then this lim inf would be constant with probabilit, 1. The Blumenthal zero-one law is one of the key tools in general Markov theory with a number of well-known uses; the zero-one laws discussed here have similar applications. In particular, when a zero-one law holds at $R$, it is often possible to decompose the given Markov process into two parts-a part uefore $R$ and a pari after $R$-which are conditionally independent given $\bar{\lambda}(R)$. Structure theorems of this type often give a good deal of insight into the functioning of the process.

Turning to a more specific des:ription of the contents of the paper, suppose that $X$ is a real stochastic process with stationary independent increments. Let $R$ be a random time and $M$ the time point on $[0, R)$ at which the process assumes a minimum; assume $P\{M<R\}>0$. Then (Theorem 3.1) conditional on $\{M<R\}$, the sigma field $\Re_{0_{+}}^{\prime}$ is trivial. Thus, unlike the case of last exit from a set, a zero-one law always holds at $M$. The existence of such a law at the minimum is closely connected with the fact that the sample function behavior at the time of the mini. num is "pure": either all the paths of the process are continuous at $M$; or else all jump into the minimum and leave continuously (i.e. $X(M-)>X(M)$ with probability 1); or else all enter the minimum continuously but jump out $(X(M-)<X(M)$ with probability 1$)$. In brief, these processes leave the minimum in only one "way". The precise criteria for which processes exhibit which behavior are given in $\$ 3$.

Suppose next that $X$ is a right continuous strong Markov process which attains its minimum strictly before its lifetime. Then, with no other hypotheses, it turns out that, conditional $I$, the value of the minimum, the post-minimum process $\{X(M+t), t>0\}$ is a Markov process with stationary transition functions which depend on $I$. A bit more precisely, if $\mathscr{F}(M+s)$ is an appropriate sigma field associated with the random time $M+s$, then for bounded Borel $f$,

$$
E\{f(X(M+t)) \mid \mathcal{F}(M+s)\}=: H_{t-s}(I ; X(M+s), f), \quad 0<s<t,
$$

where $H_{t}(a ; x, d y)$ is, for each $a$, a transition semigroup closely connected with that of $X$; see Proposition 4.1. If, in addition, a zero-one law holds at $M$, this can be strengthened to assert that the post- $M$ process depends on the events before $M$ only through $(I, X(M)$ ) (in general both of these variables are involved); see Proposition 4.2 for the precise statement. It then follows from results discussed in the previous paragraph (and in more detail in \$3) that any 
strong Markov process whose paths agree with those of a Lévy process up to a random time $R$ (such as processes obtained from Lévy processes via multiplicative and additive functionals) possess this very strong decomposition at $M$. It is also not difficult to show that any right continuous, left limit strong Markov process with no upward jumps $\left(P\left\{X_{t} \leqslant X_{t-}\right.\right.$ all $\left.\left.t\right\}=1\right)$ has a zero-one law at $M$, and so such a decomposition holds for these processes as well. This last result contains the decompositions obtained, by different methods, for certain regular diffusions by Jacobsen [11] and Williams [22].

It turns out that if $L$ is the last time that a strong Markov process leaves the interval $(-\infty, a]$, then the post- $L$ process $\{X(L+t), t>0\}$ is Markov with the same transitions as the post-minimum process (given that the value of the minimum is $a$ ). The transitions have the intuitive interpretation as those of the original process "conditioned to remain above $a$," a notion that is m: de precise by the usual Doob $h$-transform. Roughly speaking, the similarity between the two processes is due to the fact that $M$ can be viewed as the last exit from a random interval. This similarity notwithstanding, a zero-one law often fails at $L$. This phenomenon is due to the fact that at last exit-unlike the minimum-the sample function behavior often is not "pure": there are Lévy processes such that some of the paths leave $(-\infty, a]$ at $L$ continuously, and some of the paths leave by jumping out. See Proposition 5.1 for the precise criteria for this behavior. Thus, unlike paths at a minimum, the paths at a last exit can leave in more than one "way", and under such circumstances a zeroone law fails. It turns out that this is the worst that can happen-either all paths leave $(-\infty, a]$ continuously, or else all leave by jumping, or else both possibilities occur. In the first two cases a zero-one law holds; in the latter it fails. Even in the exceptional case, things are still rather nice: conditional on the mode of leaving $(-\infty, a]$, a zero-one law will still hold!

To see that continuity is not an essential ingredient making for the existence of zero-one laws consider the following example. Let $\{X(t), t \geqslant 0\}$ be Brownian motion, started at 0 , and killed on leaving $(-1,1)$; let $K_{0}$ be the last time that $X$ was zero. Evidently a zero-one law fails at $K_{0}$ since half of the paths from $K_{0}$ go to 1 and half of the paths to -1 . However, if the Brownian motion were killed on leaving $(-\infty, 1)$ and $K_{0}$ is the time of the last zero, then a zeroone law holds at $K_{0}$. Thus exit from a point introduces a further complication not present in the case of the minimum nor in the case of last exit from a halfline-the existence of a zero-one law at $K_{0}$ will depend on the nature of the random time before which $K_{0}$ is the last 0 ! Intuitively Brownian motion killed on leaving $(-1,1)$ can exit from 0 (for the last time) in two "ways", but Brownian motion killed at $\{1\}$ can exit from 0 in only one "way". While the use of minima is a key tool in the study of the last exit from $(-\infty, a]$, study of the last exit from (say) a point involves a different approach and so will be discussed elsewhere. However, once one has seen the possibility of leaving a 
set for the last time continuously but in "moie than one way" the fact that Lèvy and other Markov processes leave the minimuni "in only one way" appears quite a bit more remarkable than at first sight.

82 of this paper contains a description of basic notations together with a summary of prerequisite facts concerning the local minima of a process with independent increments. $\$ \S 3$ and 4 are devoted to the existence of zero-one laws at local minima and to the basic decompositions into pre- and postminimum processes respectively. In $\$ 5$ local zero-one laws are proved at last exit times from an interval. The proofs of the results in $\$ 5$ are, to a certain extent, more complicated variants of the methods of 83 ; accordingly the exposition of $\$ 5$ is more brief

ACKNowledgment. It is a $p$ easure to thank J. W. Pitman for several stimulating conversations on the subject mat ter this paper, and for making available to me, long before jublication, the pacers of Jacobsen and Williams.

2. Preliminaries. The basic process throughout $\S \S 3$ and 5 is a real valued process $X=\{X(t), t \geqslant 0\}$ with stationary independent increments. A familiar fact is that

$$
E \exp \{i u X(t)\}=\exp \{t \psi(u)\}
$$

where $\psi(u)=i a u-\left(\sigma^{2} / 2\right) u^{2}+\int\left[e^{i u x}-1-i u x /\left(1+x^{2}\right)\right] \nu(d x)$. The measure $v$ is called the Lévy measure, and $\psi$ is called the exponent. If $\sigma^{2}>0, X$ is said to have a Gaussian component. In those cases where $\sigma^{2}=0$, the assumption throughout is that $\nu\{(-\infty, \infty)\}=\infty$, since the problems of this paper are relatively easily solved by other methods in the compound Poisson case. Assume further, as is custon ary, that a version of $X$ has been constructed with right continuous paths, left limits, so that $X$ is strong Markov. Following current usage, we call $X$ a Lévy process.

Notations and terminology belonging to the general theory of Markov processes will, for the most part, follow those of Blumenthal and Getoor [3]. In particular, $E^{x}$ and $P^{x}$ will denote expectation and probability for the process starting at $x$; if $x=0$, the superscript will be omitted. The notation $\sigma\{\cdots\}$ will stand for the sigma field generated by whatever appears between the braces. Special sigma fields used throughout will be $\mathscr{F}_{t}^{0}=\sigma\left\{X_{s}, s<t\right\}$, $\mathscr{F}^{0}$ $=V \mathscr{F}_{t}^{0}$ and $\mathscr{F}_{t}, \mathscr{F}$ which are the usual completions of $\mathscr{F}_{t}^{0}, \mathscr{F}^{0}$ in the general theory; see [3]. As usual, notations $X_{t}, X(t) ; \mathscr{F}_{t}, \mathscr{F}(t)$; and so forth will be used interchangeably according to typographical convenience.

Let $P_{t}(x, d y)$ be the transition functions of the Lévy process $X$. If $\mu_{t}(d y)$ denotes the $P^{0}$ distribution of $X_{t}$ then $P_{t}(x, d y)=\mu_{t}(d y-x)$. If $\hat{\mu}_{t}(d y)$ is the $P^{0}$ distribution of $-X_{t}$, then the transition functions $\hat{P}_{t}(x, d y)=\hat{\mu}_{t}(d y-x)$ are in duality with those of $X$ in the sense that $\left\langle P_{t} f, g\right\rangle=\left\langle f, P_{t} g\right\rangle$. Expectation and probability on $\Omega$ corresponding to $\hat{P}_{t}(x, d y)$ will be denoted by $\hat{E}^{x}, \hat{P}^{x}$. In 
using duality we will always work with the canonical (i.e. function space) representation of the processes involved; in this case both the original process and its dual $X$ are just coordinate maps on $\Omega$, so $X_{t}(\omega)=X_{t}(\omega)$.

A random time $R$ is a nonnegative function on the basic probability space, measurable with respect to $\mathscr{F}$. Often $R$ will be defined only on part of the probability space; the definition can be completed by putting $R=\infty$ on the remaining part of the space. However, the random variables $X(R)$ will be considered only on $\{\omega: R(\omega)$ is defined and finite $\}$. The process $\{X(R+t), t$ $>0\}$ will be called the post- $R$ process. Several sigma fields involving random times arise frequently. The definitions to follow are for any right continuous Markov process relative to right continuous, complete sigma fields $\mathcal{F}_{t}$. The strict past of $R$, denoted by $\mathscr{F}(R-)$ is defined by

$$
\mathscr{F}(R-)=\sigma\left\{A \cap\{R>t\}: t>0, A \in \mathscr{F}_{t}\right\} .
$$

It is easy to see that for random times $R \leqslant T$

(2.3) (a) $\mathscr{F}(R-) \subset \mathscr{F}(T-)$ if $R \in \mathscr{F}(T-)$.

(b) $\mathscr{F}\{(R+t)-\}, t \geqslant 0$ is an increasing family of sigma fields.

(c) $R$ is $\mathscr{F}(R-)$-measurable.

(d) If the paths of $X$ have left limits, $X(R-)$ is $\mathscr{F}(R-)$ measurable, where $X(R-)$ is defined only on $\{0<R<\infty\}$.

The second sigma field associated with $R$ is

(2.4) $\mathscr{F}(R)=\left\{A \in \mathscr{F}_{:}\right.$for all $t \geqslant 0$, there exists $A_{t} \in \mathscr{F}_{t}$ such that $A$ $\left.\cap\{R<t\}=A_{t} \cap\{R<t\}\right\}$. This sigma field was introduced in [15]. It is easy to check that

(2.5) (a) If $R \leqslant T, \mathscr{F}(R) \subset \mathscr{F}(T)$.

(b) If $R_{n} \downarrow R, \mathscr{F}(R)=\cap \mathscr{F}\left(R_{n}\right)$; in particular, $\{\mathscr{F}(R+t), t \geqslant 0\}$ is right continuous.

(c) $X(R)$ is $\mathscr{F}(R)$-measurable if and only if, for every $t, X(R)$ is equal on $\{R<t\}$ to some $\mathscr{F}_{t}$-measurable random variable.

Finally, it will often be necessary to discuss the sigma fields

$$
\Re_{t}=\sigma\{X(R+s)-X(R), s \leqslant t\} \text { and } \Re_{0+}=\bigcap_{i 0} \Re_{t} .
$$

Because of conventions introduced before, such sigma fields consist of subsets of $\{\omega: R$ is defined and finite $\}$.

The remainder of this section summarizes several facts about the local minima of a Lévy process that will be needed in $\$ \S 3$ and 4 . Let

$$
I_{t}=\inf _{s<t} X_{s} .
$$

Since $X$ has only moving discontinuities, $I_{t}=\inf _{s<t} X_{s}$ a.s. Recall that 0 is regular for a set $A$ if $P\left\{T_{A}=0\right\}=1$, where $T_{A}=\inf \left\{t>0: X_{t} \in A\right\}$. For the 
Lévy processes considered here, 0 is regular for at least one of the intervals $(-\infty, 0),(0, \infty)$ and often is regular for both. The precise criteria for which this holds can be found in Rogozin [20].

Proposition 2.1. (a) If $t>0, P\left\{I_{t}=X_{t}\right\}>0$ if and only if 0 is not regular for $(0, \infty)$.

(b) $P\left\{I_{t}=0\right\}>0$ if and only if 0 is not regular for $(-\infty, 0)$.

In particular, if 0 is regular for $b$ th $(-\infty, 0)$ and $(0, \infty)$, then the minimum of $X$ on $[0, t]$ falls in the interior of the interval.

Proor. By duality,

$$
P\left\{I_{t}=X_{t}\right\}=P\left\{X_{s} \leqslant 0, \text { all } s \leqslant t\right\}>0
$$

if 0 is not regular for $(0, \infty)$ and this proves (a). Part (b) is obvious.

A real right continuous funcion $f$ on $[a, b]$ having left limits will be said to attain its minimum at a point $M \in[a, b]$ if either $f(M)=\inf \{f(t): t \in[a, b]\}$ or $f(M-)=\inf \{f(t): t \in[a, b]\}$.

Proposition 2.2. On each interval $(a, b)$, almost every path of $X$ attains its minimum at exactly one point of $[a, b]$.

Proof. The closures of the sets

$$
A_{1}=\left\{(t, x): X_{t}=x<X_{s}, s<t\right\}
$$

and

$$
A_{2}=\left\{(t, x): X_{t}=x \leqslant X_{s}, s \leqslant t\right\}
$$

coincide a.s. with the closed ranges of two 2-dimensional subordinators $\tau_{1}, \tau_{2}$ respectively. These two subordinators are the same when $X$ is not compound Poisson (Fristedt [6, Theorem 9.1 and Corollary 9.2]), whereupon the proposition is evident.

Let

(2.7) $M_{t}$ be the unique point of $[0, t]$ where $X$ assumes its minimum value.

Then the next proposition is easy.

Proposition 2.3. Suppose that neither $X$ nor $-X$ is a subordinator. Then $P\left\{0<M_{t}<t\right\}>0$.

The definitive version of the next proposition appears in $\$ 3$.

Proposition 2.4. (a) If 0 is regular for $(0, \infty)$, then $X\left(M_{t}\right)=I_{t}$.

(b) If 0 is regular for $(-\infty, 0)$ then $X\left(M_{t}-\right)=I_{t}$.

Remark. Of course, if 0 is regular for $(0, \infty)$ and for $(-\infty, 0)$ then $X$ is 
continuous at $M_{t}$. The question whether $X\left(M_{t}\right)=X\left(M_{t}-\right)$ is possible in the case where (say) 0 is regular for $(-\infty, 0)$ but not $(0, \infty)$ will be answered (negatively) in $\$ 3$.

Proof. Suppose that 0 is regular for $(-\infty, 0)$. If $X\left(M_{t}-\right)>X\left(M_{t}\right)$ on part of $\left\{0<M_{t}<t\right\}$, then the minimum on $[0, t]$ occurs at a time at which $X$ jumps. But by the strong Markov property applied to jump times and the assumed regularity of 0 for $(-\infty, 0)$, the process upon completing any jump immediately goes below the point to which it just jumped. It follows in this case that it is impossible that $X\left(M_{t}-\right)>X\left(M_{t}\right)$ on $\left\{0<M_{t}<t\right\}$ proving (b). Next suppose 0 is regular for $(0, \infty)$. Let

$$
\begin{aligned}
& A=\left\{\omega: 0<M_{t}<t, X(M-)<X(M)\right\}, \\
& \hat{A}=\left\{\omega: 0<M_{t}<t, X(M-)>X(M)\right\} .
\end{aligned}
$$

If $\xi$ is Lebesgue measure, then an extension (Walsh [21]) of the basic duality relation implies that

$$
P^{\xi}(A)=\hat{P}^{\xi}(\hat{A}) .
$$

However if 0 is regular for $(0, \infty)$ (for $X$ ), then 0 is regular for $(-\infty, 0)$ for the dual process, so the first part of the proof forces $\hat{P}^{\xi}(\hat{A})=0$. Hence $P^{x}\{A\}$ $=0$ for almost all $x$, and hence for all $x$ by translation invariance.

3. Zero-one law at the minimum. Let $X=\left\{X_{t}, t \geqslant 0\right\}$ again be a real Lévy process and let $S=S^{\lambda}$ be a random variable independent of $X$ such that $P\{S>t\}=e^{-\lambda t}(\lambda>0)$. If $\lim _{t \rightarrow \infty} X_{t}=+\infty$, then $\lambda=0$ will also be allowed in the arguments to follow. Let $X^{\lambda}=\left\{X_{t}^{\lambda}, t \geqslant 0\right\}$ be defined by

$$
\begin{aligned}
X_{t}^{\lambda} & =X_{t}, t<S, \\
& =\infty, t \geqslant S,
\end{aligned}
$$

and define $I^{\lambda}=I$ by

$$
I^{\lambda}=\inf _{s} X_{s}^{\lambda}
$$

Let $M=M^{\lambda}$ be the unique time point at which $X^{\lambda}$ attains its minimum; either $X(M)=I$ or $X(M-)=I$. Let $\mathscr{F}_{t}^{\lambda}$ be the usual completion of the sigma field $\sigma\left\{X_{s}^{\lambda}, s \leqslant t\right\}$ and define $\sigma^{\lambda}(M-)$ in the obvious way (see (2.4)). The first proposition shows that the post-minimum process $\left\{X^{\lambda}(M+t), t\right.$ $>0$ ) depends only on $X(M-)$ and $X(M)$; it will be clear that $X^{\lambda}(M-)$ is involved only if $P\left\{X^{\lambda}(M)>X^{\lambda}(M-), M<S\right\}>0$ (i.e. if 0 is not regular for $(0, \infty))$. Recall that a subordinator is a Lévy process with nondecreasing paths; criteria for $X$ to be a subordinator are well known (see [7]). 
Proposmon 3.1. Assume that $-X$ is not a subordinator. The process $\left\{X^{\lambda}(M+t)-I^{\lambda}, t>0\right\}$ is conditionally independent of $\mathscr{F}^{\lambda}(M-)$, given $S^{\lambda}$ $>M:$ if $A \in \mathcal{F}^{\lambda}(M-), B \in \sigma\left\{X^{\lambda}(M+t)-I^{\lambda}, t>0\right\}$, then

$$
P\left\{A \cap B \mid S^{\lambda}>M\right\}=P\left\{A \mid S^{\lambda}>M\right\} P\left\{B \mid S^{\lambda}>M\right\} .
$$

Proor. Since $-X$ is not a subordinator, $P\left\{S^{\lambda}>M\right\}>0$. Let $\varepsilon>0$ and define $T_{0}=\inf \left\{t>0:\left|X_{t}\right|>\varepsilon\right\}, T_{n}=\inf \left\{t>0:\left|X\left(t+T_{n}\right)-X\left(T_{n}\right)\right|>\varepsilon\right\}$, $n>1$. Let $X_{0}=0, X_{n}=X\left(T_{n}\right), n>1$. By the strong Markov property, $\left\{X_{n}, n>0\right\}$ is a random walk; $\left\{T_{n}, n \geqslant 0\right\}$ is a sequence of independent, identically distributed random variables; and $\left(X_{0}, X_{1}, \ldots, X_{n}, T_{0}, \ldots, T_{n-1}\right)$ is independent of $\sigma\left\{X_{n+j}-X_{n}, j>1 ; T_{i}, i \geqslant n\right\}$. The right continuous process $\left\{Y_{t}, t>0\right\}$ defined by

$$
\begin{aligned}
Y_{t} & =0, & & t<T_{0}, \\
& =X_{n}, & & T_{0}+\cdots+T_{n-1}<t<T_{0}+\cdots+T_{n},
\end{aligned}
$$

then converges as $\varepsilon \rightarrow 0$ pathwise, uniformly to $\left\{X_{t}, t>0\right\}$. Define the process $\left\{Y_{t}^{\lambda}, t>0\right\}$ by

$$
Y_{t}^{\lambda}=Y_{t} \text { if } t<T_{0}+\cdots+T_{N},
$$

where $N=\inf \left\{n: T_{0}+\cdots+T_{n}>S^{\lambda}\right\}$;

$$
Y_{t}^{\lambda}=\infty \text { if } t \geqslant T_{0}+\cdots+T_{N} .
$$

Then as $\varepsilon \rightarrow 0, Y^{\lambda}$ converges pathwise to $X^{\lambda}$, and uniformly for $t \in[0, S)$. Let $Q$ be the index $i$ for which $X_{i}$ is the (last) minimum of $Y^{\lambda}$, let $Y_{i}^{\lambda}, i \geqslant 0$, be the successive values of $Y^{\lambda}$, and $T_{i}^{\lambda}$ the interjump times for $Y^{\lambda}$. So if $\lambda>0$, there will exist an $i$ such that $Y_{i}^{\lambda}=T_{i}^{\lambda}=\infty$. Notice that $Y_{Q}^{\lambda}$ is finite with probability 1 and that as $\varepsilon \rightarrow 0, Y_{Q}^{\lambda}$ converges to $I^{\lambda}=\inf _{s} X_{s}^{\lambda}$. Let $A, B$, $C, D$ be Borel subsets of $(-\infty, \infty)$. Then, for example, if $i>1$

$$
\begin{aligned}
& P\left\{Y_{Q-i}^{\lambda} \in A, T_{Q-i}^{\lambda} \in B, Y_{Q+k}^{\lambda}-Y_{Q}^{\lambda} \in C, T_{Q+k}^{\lambda} \in D, N>Q>i\right\} \\
& =\sum_{l>i} P\left\{Y_{l-i}^{\lambda} \in A, T_{l-i}^{\lambda} \in B, Y_{l+k}^{\lambda}-Y_{l}^{\lambda} \in C, T_{l+k}^{\lambda} \in D, N>Q=l\right\} .
\end{aligned}
$$

Since $C, D$ do not contain $\infty$, a typical term in the summation of (3.5) may be written

$$
\begin{aligned}
\sum_{n>l+k} P\left\{Q=l, Y_{l-i} \in A,\right. & T_{l-i} \in B, Y_{l+k}-Y_{l} \in C, T_{l+k} \in D, \\
& \left.T_{0}+\cdots+T_{n-1} \leqslant S<T_{0}+\cdots+T_{n}\right\} .
\end{aligned}
$$


A typical term in the sum (3.6) may be written

$$
P\left\{Y_{l} \leqslant Y_{m} \text {, all } m \leqslant l ; Y_{l+u}>Y_{l}, 0<u<n-l ;\right.
$$

$$
\begin{array}{r}
Y_{l-i} \in A, T_{l-i} \in B, T_{0}+\cdots+T_{n-1} \leqslant S<T_{0}+\cdots+T_{n}, Y_{l+k}-Y_{l} \\
\left.\in C, T_{l+k} \in D\right\} .
\end{array}
$$

Upon replacing $T_{0}+\cdots+T_{n-1} \leqslant S<T_{0}+\cdots+T_{n}$ by $e^{-\lambda\left(T_{0}+\cdots+T_{n-1}\right)}$ $-e^{-\lambda\left(T_{0}+\cdots+T_{n}\right)}$ and using the independence structure of the $Y_{i}, T_{i}$ detailed at the beginning of the proof, (3.7) becomes

$E\left\{Y_{l} \leqslant Y_{m}, m \leqslant l ; Y_{l-i} \in A, T_{l-i} \in B, e^{-\lambda\left(T_{0}+\cdots+T_{l-1}\right)}\right\}$

$$
\times E\left\{Y_{u}>0,0<u<n-l ; e^{-\lambda\left(T_{0}+\cdots+T_{n-l-1}\right)}-e^{-\lambda\left(T_{0}+\cdots+T_{n-1}\right)} ;\right.
$$

$$
\left.Y_{k} \in C, T_{k} \in D\right\}
$$

$$
\begin{array}{r}
=P\left\{Y_{l} \leqslant Y_{m}, m \leqslant l ; Y_{l-i} \in A, T_{l-i} \in B, T_{0}+\cdots+T_{l-1}<S\right\} \\
\times P\left\{Y_{u}>0,0<u<n-l ; T_{0}+\cdots+T_{n-l-1}<S \leqslant T_{0}+\cdots+T_{n-l} ;\right. \\
\left.\quad Y_{k} \in C, T_{k} \in D\right\} .
\end{array}
$$

The result of summing (3.8) over all $n \geqslant l+k$ is

$$
\begin{aligned}
P\left\{Y_{l} \leqslant\right. & \left.Y_{m}, m \leqslant l ; Y_{l-i} \in A, T_{l-i} \in B, T_{0}+\cdots+T_{l-1}<S\right\} \\
& \times P\left\{Y_{i}^{\lambda}>0, \text { all } i>0 ; Y_{k}^{\lambda} \in C, T_{k}^{\lambda} \in D\right\} \\
= & P\left\{Y_{k}^{\lambda} \in C, T_{k}^{\lambda} \in D \mid Y_{i}^{\lambda}>0 \text { all } i>0\right\} P\left\{Y_{i}^{\lambda}>0 \text { all } i>0\right\} \\
\quad & \times P\left\{Y_{l} \leqslant Y_{m}, m \leqslant l ; Y_{l-i} \in A, T_{l-i} \in B, T_{0}+\cdots+T_{l-1}<S\right\} .
\end{aligned}
$$

The hypothesis that $-X$ is not a subordinator guarantees that $P\left\{Y_{i}^{\lambda}>0\right.$ all $i>0\}>0$. If $\Gamma$ denotes the last factor in (3.9), then the last two factors after the equality in (3.9) may be written

$$
\begin{array}{rl}
\sum_{j} & P\left\{Y_{i}^{\lambda}>0, i>0 ; T_{0}+\cdots+T_{j-1} \leqslant S<T_{0}+\cdots+T_{j}\right\} \Gamma \\
& =\sum_{j} P\left\{Y_{i}>0,0<i<j ; e^{-\lambda\left(T_{0}+\cdots+T_{j-1}\right)}-e^{-\lambda\left(T_{0}+\cdots+T_{j}\right)}\right\} \Gamma \\
& =\sum_{j} P\left\{Y_{i}>Y_{l}, l<i<j+l ; e^{-\lambda\left(T_{l}+\cdots+T_{l+j-1}\right)}-e^{-\lambda\left(T_{l}+\cdots+T_{l+j}\right)}\right\} \Gamma \\
& =\sum_{j} P\left\{Q=l, Y_{l-i} \in A, T_{l-i} \in B, T_{0}+\cdots+T_{j+l-1}\right. \\
& \left.\quad<S \leqslant T_{0}+\cdots+T_{j+l}\right\} \\
= & P\left\{Q=l<N, Y_{l-i}^{\lambda} \in A, T_{l-i}^{\lambda} \in B\right\} .
\end{array}
$$


Substitute the result of (3.10) into (3.9) and sum over $l>i$ to obtain

$$
\begin{gathered}
P\left\{Y_{Q-i}^{\lambda} \in A, T_{Q-i}^{\lambda} \in B, Y_{Q+k}^{\lambda}-Y_{Q}^{\lambda} \in C, T_{Q+k}^{\lambda} \in D, N>Q \geqslant i\right\} \\
=P\left\{Y_{k}^{\lambda} \in C, T_{k}^{\lambda} \in D \mid Y_{n}^{\lambda}>0 \text { all } n>0\right\} \\
\times P\left\{N>Q \geqslant i, Y_{Q-i}^{\lambda} \in A, T_{Q-i}^{\lambda} \in B\right\} .
\end{gathered}
$$

Since the first factor in (3.11) after the equality does not depend on $i, A, B$, set $A=B=(-\infty, \infty)$ to see that

$$
\begin{aligned}
P\left\{Y_{k}^{\lambda} \in C, T_{k}^{\lambda}\right. & \left.\in D \mid Y_{i}^{\lambda}>0, i>0\right\} \\
& =P\left\{Y_{Q+k}^{\lambda}-Y_{Q}^{\lambda} \in C, T_{Q+k}^{\lambda} \in D, N>Q\right\}
\end{aligned}
$$

whereupon

$$
\begin{aligned}
& P\left\{Y_{Q-i}^{\lambda} \in A, T_{Q-i}^{\lambda} \in B, Y_{Q+k}^{\lambda}-Y_{Q}^{\lambda} \in C, T_{Q+k}^{\lambda} \in D, N>Q>i\right\} \\
&= P\left\{Y_{Q+k}^{\lambda}-Y_{Q}^{\lambda} \in C, T_{Q+k}^{\lambda} \in D, N>Q\right\} \\
& \times P\left\{Y_{Q-i}^{\lambda} \in A, T_{Q-i}^{\lambda} \in B, N>Q \geqslant i\right\} .
\end{aligned}
$$

A similar analysis gives the same result if $C, D$ contain $+\infty$. Of course, the same argument works if one considers instead

$$
\begin{array}{r}
P\left\{Y_{Q-i_{j}}^{\lambda} \in A_{j}, T_{Q-i_{j}}^{\lambda} \in B_{j}, Y_{Q+k_{j}}^{\lambda}-Y_{Q}^{\lambda} \in C_{j}, T_{Q-k_{j}}^{\lambda} \in D_{j},\right. \\
j=1,2, \ldots, n, N>Q\},
\end{array}
$$

and this shows that the pre- $Q$ process $\left\{Y_{t}^{\lambda}, t<Q\right\}$ is conditionally independent of $\left\{Y^{\lambda}(t+Q)-Y^{\lambda}(Q), t \geqslant 0\right\}$, given $Q<N$. A standard limiting argument as $\varepsilon \rightarrow 0$ shows $\left\{X^{\lambda}(t+M)-I^{\lambda}, t>0\right\}$ is conditionally independent of $\mathscr{F}(M-)$, the conditioning involved being the elementary one of beginning probability theory.

The next lemma is the key step in the proof of the zero-one law at the minimum. The notation is that of Proposition 3.1.

Lemma 3.1. Assume that $-X$ is not a subordinator. Let $\delta>0$ and let $M_{\delta}=M_{\delta}^{\lambda}$ be the time point (if any) where $\left\{X_{s}^{\lambda}, s>M^{\lambda}+\delta\right\}$ assumes its minimum. Let $I_{\delta}=I_{\delta}^{\lambda}$ be the value of this minimum. Then $\left\{X^{\lambda}\left(M_{\delta}+t\right)\right.$ $\left.-X^{\lambda}\left(M_{\delta}\right), t>0\right\}$ is conditionally independent of $\mathscr{F}^{\lambda}\left(M_{\delta}-\right)$, given $\left\{S^{\lambda}>M^{\lambda}\right.$ $+\delta\}$.

An interesting corollary follows from the proof.

Corollary 3.1. Conditional on $M+\delta<S$, the process $\left\{X^{\lambda}\left(M_{\delta}+t\right)\right.$ $\left.-X^{\lambda}\left(M_{\delta}\right), t \geqslant 0\right\}$ has the same distribution as $\left\{X^{\lambda}(M+t)-X^{\lambda}(M), t \geqslant 0\right\}$. 
Proof. For $\varepsilon>0$, define $\left\{Y_{t}, t \geqslant 0\right\},\left\{Y_{t}^{\lambda}, t \geqslant 0\right\},\left\{Y_{i}\right\},\left\{Y_{i}^{\lambda}\right\},\left\{T_{i}\right\},\left\{T_{i}^{\lambda}\right\}$ and $Q$ as in the proof of Proposition 3.1. Set

$$
\begin{aligned}
N(\delta)= & \min \left\{i: T_{Q+1}+\cdots+T_{i} \geqslant \delta\right\}, \quad R=T_{0}+\cdots+T_{N(\delta)}, \\
Q(\delta)= & \text { the index } i \text { for which } Y_{i}^{\lambda} \text { is the (last) minimum of } \\
& \left\{Y_{t}^{\lambda}, t>R\right\} \quad(\text { defined only on } R<S) .
\end{aligned}
$$

If $A, B, C, D$ are Borel sets as in the proof of Proposition 3.1

$$
\begin{gathered}
P\left\{Y_{Q(\delta)-i}^{\lambda} \in A, T_{Q(\delta)-i}^{\lambda} \in B, Y_{Q(\delta)+k}^{\lambda}-Y_{Q(\delta)}^{\lambda} \in C,\right. \\
\left.T_{Q(\delta)+k}^{\lambda} \in D, Q(\delta)>i, S>R\right\} \\
=\sum_{\substack{l>i \\
u<l}} P\left\{Y_{l-i}^{\lambda} \in A, T_{l-i}^{\lambda} \in B, Y_{l+k}^{\lambda}-Y_{l}^{\lambda} \in C,\right. \\
\left.T_{l}^{\lambda} \in D, Q(\delta)=l, Q=u, S>R\right\} .
\end{gathered}
$$

A typical term in the sum (3.14) can be written

$$
\begin{gathered}
\sum_{l>b>u} P\left\{Y_{l-i}^{\lambda} \in A, T_{l-i}^{\lambda} \in B, Y_{l+k}^{\lambda},-Y_{l}^{\lambda} \in C,\right. \\
\left.T_{l+k}^{\lambda} \in D, Q(\delta)=l, Q=u, S>T_{l}+\cdots+T_{b}, N(\delta)=b\right\} .
\end{gathered}
$$

Using the definition of $N(\delta)$, a typical term in (3.15) can be written (since $C$, $D$ do not contain $\infty$ ):

$$
\begin{array}{r}
\sum_{p>l+k} P\left\{Y_{l-i} \in A, T_{l-i} \in B, Y_{l+k}-Y_{l} \in C, T_{l+k} \in D, Q(\delta)=l, Q=u\right. \\
T_{0}+\cdots+T_{p-1} \leqslant S<T_{0}+\cdots+T_{p}, T_{u+1}+\cdots+T_{b} \geqslant \delta \\
\left.>T_{u+1}+\cdots+T_{b-1}\right\} .
\end{array}
$$

Finally, a typical term of (3.16) may be written, using the definitions of $Q(\delta)$ and $Q:$ for $l, u, p, b$ with $l>i, u<l, u<b<l, p \geqslant l+k$,

$$
\begin{array}{r}
P\left\{Y_{l-i} \in A, T_{l-i} \in B, Y_{l+k}-Y_{l} \in C, T_{l+k} \in D,\right. \\
T_{u+1}+\cdots+T_{b} \geqslant \delta>T_{u+1}+\cdots+T_{b-1}, T_{0}+\cdots+T_{p-1} \leqslant S \\
<T_{0}+\cdots+T_{p}, Y_{m} \geqslant Y_{u}, m<u ; Y_{m}>Y_{u}, u<m \leqslant p, Y_{l} \leqslant Y_{r}, \\
\left.\quad b<r<l ; Y_{l}<Y_{r}, l<r<p\right\} .
\end{array}
$$

Since $Y_{l}>Y_{u}$, the inequalities in (3.17) involving $Y_{m}, Y_{u}, Y_{l}, Y_{r}$ may be replaced by 


$$
\begin{array}{ccc}
Y_{m} \geqslant Y_{u}, \quad m<u ; & Y_{m}>Y_{u}, \quad u<m \leqslant b, \\
Y_{r} \geqslant Y_{l}, \quad b<r<l ; & Y_{l}<Y_{r}, \quad l<r<p .
\end{array}
$$

As in the proof of Proposition 3.1, an application to (3.17) of the independence properties of the $\left\{Y_{i}\right\}$ and $\left\{T_{i}\right\}$ and a summation over $l, u, b, p$ of the result yields

$$
\begin{aligned}
P\left\{Y_{Q(\delta)-i}^{\lambda}\right. & \left.\in A, T_{Q(\delta)-i}^{\lambda} \in B, Y_{Q(\delta)+k}^{\lambda} \in C, T_{Q(\delta)+k}^{\lambda} \in D, S>R\right\} \\
= & P\left\{Y_{Q(\delta)-i}^{\lambda} \in A, T_{Q(\delta)-i}^{\lambda} \in B, S>R\right\} \\
& \times P\left\{Y_{Q(\delta)+k}^{\lambda} \in C, T_{Q(\delta)+k}^{\lambda} \in D \mid S>R\right\} .
\end{aligned}
$$

Here again $P\{S>R\}>0$ since $-X$ is not a subordinator. Extension as before and passage to the limit as $\varepsilon \downarrow 0$ leads to the desired conclusion.

The next lemma establishes the zero-one law in a special case and, in addition, settles an issue remaining open from Proposition 2.4. With the notation the same as in Proposition 3.1, define

$$
\begin{aligned}
\mathscr{F}_{t}^{\prime} & =\sigma\left\{X^{\lambda}\left(M^{\lambda}+s\right)-X^{\lambda}\left(M^{\lambda}\right), 0<s<t\right\}, \\
\mathscr{F}_{0+}^{\prime} & =\bigcap_{t>0} \mathscr{F}_{t}^{\prime}, \\
B & =\left\{\omega: X^{\lambda}\left(M^{\lambda}\right)=I^{\lambda}\right\} .
\end{aligned}
$$

LEMMA 3.2. (a) $P\{B \mid M<S\}=1$ or 0 according as 0 is regular for $(0, \infty)$ or not; in particular if 0 is not regular for $(0, \infty), X^{\lambda}$ always jumps out of its ultimate minimum.

(b) If $A \in \mathscr{F}_{0_{+}^{\prime}}^{\prime}$, then $P\{A \mid S>M\}=0$ or 1 .

Proof. Let $\delta>0$ and $A \in \mathscr{F}_{0+}^{\prime}$. Then with the notation of Lemma 2.1, $A \cap\left\{S>M_{\delta}\right\} \in \mathscr{F}\left(M_{\delta}-\right)$ so $A \cap\left\{S>M_{\delta}\right\}$ is conditionally independent of $\sigma\left\{X^{\lambda}\left(M_{\delta}+u\right)-I_{\delta}^{\lambda}, u>0\right\}$, given $S>M+\delta$. Also, if

$$
\mathscr{F}_{t}^{\prime \prime}=\sigma\left\{X^{\lambda}(M+u)-I^{\lambda}, 0 \leqslant u \leqslant t\right\}, \quad \mathscr{F}_{0+}^{\prime \prime}=\bigcap_{t>0} \mathscr{F}_{t}^{\prime \prime},
$$

then $B \in \mathscr{F}_{0_{+}}^{\prime \prime}$ and so $A \cap B$ is also conditionally independent of

$$
\sigma\left\{X^{\lambda}\left(M_{\delta}+u\right)-I_{\delta}^{\lambda}, u>0\right\}
$$

given $\{S>M+\delta\}$. Hence, for example

$$
P\left\{A B C_{\delta} \mid S>M+\delta\right\}=P\{A B \mid S>M+\delta\} P\left\{C_{\delta} \mid S>M+\delta\right\}
$$

where 


$$
C_{\delta}=\left\{X^{\lambda}\left(M_{\delta}+t_{i}\right)-I_{\delta}^{\lambda}<r_{i}, i=1,2, \ldots, n\right\}
$$

for a fixed sequence $0<t_{1}<\cdots<t_{n}$ and fixed real numbers $r_{1}, \ldots, r_{n}$. Let $\delta \downarrow 0$. Then on $B, X^{\lambda}\left(M_{\delta}+t\right)-I_{\delta}^{\lambda}$ converges to $X^{\lambda}(M+t)-X^{\lambda}(M)$, while in general on $M<S$,

$$
M_{\delta} \downarrow M^{*} \geqslant M, \quad I_{\delta}^{\lambda} \downarrow I^{*} \geqslant I^{\lambda}
$$

and so in general $X^{\lambda}\left(M_{\delta}+t\right)-X^{\lambda}\left(M_{\delta}\right)$ converges to a process $X^{\lambda}\left(M^{*}+t\right)$ $-I^{*}$ which, since the distributions do not change with $\delta$ (see Corollary 3.1), has the same distribution as $X^{\lambda}(M+t)-I^{\lambda}$. It follows from this and (3.21) that, if $P\{B, S>M\}$ is supposed positive,

$$
P\left(B A C_{0}, S>M\right) / P(A B, S>M)=P\left(C_{0}, S>M\right) / P(S>M)
$$

where $M_{0}=M$ when $\delta=0$; and this in turn yields

$$
P\left(B C_{0}, S>M\right) / P(B, S>M)=P\left(C_{0}, S>M\right) / P(S>M) \text {. }
$$

The equality (3.23) persists for all $C_{0} \in V \mathscr{F}_{\prime}^{\prime \prime}$ since sets of the form (3.22) with $\delta=0$ generate this sigma field. But $B \in V_{l>0} \mathscr{F}_{t \prime}^{\prime \prime}$, so choosing $C_{0}=B$ in (3.22) forces $P(B \mid S>M)=1$. Suppose now that 0 is not regular for $(0, \infty)$, It is easy to see that $P\left\{X^{\lambda}(M)>I^{\lambda}\right\}>0$. Indeed by a duality argument (see proof of Proposition 2.4) it is enough to show that a Lévy process having 0 not regular for $(-\infty, 0)$ attains its minimum by jumping (with positive probability), and this may be shown by an argument very similar to that of [16, Proposition 2.1]. This being so it now follows from what has just been proved that $P\left\{X^{\lambda}(M)>I^{\lambda} \mid M<S\right\}=1$. This completes the proof of part (a) of Lemma 3.2, since if 0 is regular for $(0, \infty), X^{\lambda}\left(M^{\lambda}\right)=I^{\lambda}$ by Proposition 2.4.

Turning to part (b), assume first that 0 is regular for $(0, \infty)$. By part (a), (3.21) becomes $P\left\{A C_{\delta} \mid S>M+\delta\right\}=P\{A \mid S>M+\delta\} P\left\{C_{\delta} \mid S>M+\delta\right\}$. Let $\delta \downarrow 0$ and this becomes $P\left\{A C_{0} \mid S>M\right\}=P\{A \mid S>M\} P\left\{C_{0} \mid S>M\right\}$ which persists for all $C_{0} \in V_{t} \mathscr{F}_{t}^{\prime \prime}$. The substitution of $A$ for $C_{0}$ leads immediately to the desired conclusion. Finally, if 0 is not regular for $(0, \infty)$, then the process always jumps out of its minimum (if it occurs before $S$ ) by part (a). Let $J_{1}, J_{2}, \ldots$ be an enumeration of the jump times of $X$. By the strong Markov property and the Blumenthal zero-one law,

$$
\mathscr{K}_{n}=\bigcap_{t>0} \sigma\left\{X\left(J_{n}+s\right)-X\left(J_{n}\right), 0 \leqslant s \leqslant t\right\}
$$

is trivial, and so the result follows from

$$
\mathscr{F}_{0+} \cap\{S>M\} \subset\left(\underset{n}{\vee} \mathscr{K}_{n}\right) \cap\{S>M\}
$$


The next theorem, which localizes the conclusions of Lemma 3.2, is the main result of this section:

Theorem 3.1. Let $X=\left\{X_{t}, t \geqslant 0\right\}$ be a real Lévy process with $-X$ not a subordinator. Let $R$ be a random time, let $I=\inf _{0<s<R} X_{s}$, and let $M$ be the point in $[0, R]$ where this minimum is achieved. Assume that $P\{M<R\}>0$. Let $\mathscr{R}=\bigcap_{t>0} \sigma\{X(M+u)-X(M), 0 \leqslant u \leqslant t\}$. Then:

(a) $P\{X(M)=I \mid M<R\}=1$ if 0 is regular for $(0, \infty)$.

(b) $P\{X(M)>I \mid M<R\}=1$ if 0 is not regular for $(0, \infty)$.

(c) If $A \in \Re, P\{A \mid M<R\}=0$ or 1 .

Remark. By time reversal, $P\{X(M)=I>X(M-) \mid 0<M<R\}=1$ if 0 is regular for $(0, \infty)$ but not for $(-\infty, 0)$.

Proof. Let $S^{\lambda}, \lambda$ rational and positive, be a family of independent random variables, independent of $X$, with $P\left\{S^{\lambda}>t\right\}=e^{-\lambda t}$. Then for almost every $\omega$ the set $\left\{S^{\lambda}(\omega), \lambda>0\right\}$ is dense on $[0, \infty)$. Let $X^{\lambda}, I^{\lambda}, M^{\lambda}$ be defined as usual. Then in case (a),

$$
\{X(M)<I, M<R\} \subset \bigcup_{\lambda}\left\{X^{\lambda}\left(M^{\lambda}\right)<I^{\lambda}, M^{\lambda}<S^{\lambda}\right\}
$$

and the latter has probability zero by Lemma 3.2. Hence $P\{X(M)=I, M$ $<R\}=P\{M<R\}$, proving (a). The proof of (b) is similar. As for (c), let $A \in \mathfrak{N}$. Then $A \in \sigma\{X(M+s)-X(M), s \leqslant 1 / n\}$ so there exists a Borel function $f_{n}$ on $R^{\infty}$, the countable product space of the real line, and time points $\left\{t_{n, k}: k \geqslant 1\right\}, t_{n, k} \leqslant n^{-1}$ with

$$
A=f_{n}\left(X\left(M+t_{n 1}\right)-X(M), X\left(M+t_{n 2}\right)-X(M), \ldots\right)
$$

and

$$
A=\limsup _{n \rightarrow \infty} f_{n}\left(X\left(M+t_{n 1}\right)-X(M), \ldots\right) .
$$

But on $S^{\lambda}>R>M^{\lambda}$,

$$
X\left(M^{\lambda}+t\right)=X(M+t)
$$

for all sufficiently small $t$, so on $S^{\lambda}>R>M^{\lambda}$,

$$
A=\limsup _{n \rightarrow \infty} f_{n}\left(X\left(M^{\lambda}+t_{n 1}\right)-X\left(M^{\lambda}\right), \ldots\right) \equiv A^{\lambda} .
$$

Of course $A^{\lambda} \in \cap_{t>0^{\sigma}}\left\{X\left(M^{\lambda}+s\right)-X\left(M^{\lambda}\right), s<t\right\}$ so $P\left(A^{\lambda}\right)=0$ or else $P\left\{A^{\lambda}, S^{\lambda}>R>M^{\lambda}\right\}=P\left\{S^{\lambda}>R>M^{\lambda}\right\}$. Suppose the latter alternative holds (otherwise consider the complement); then

$$
P\left\{A, S^{\lambda}>R>M^{\lambda}\right\}=P\left\{A^{\lambda}, S^{\lambda}>R>M^{\lambda}\right\}=P\left\{S^{\lambda}>R>M^{\lambda}\right\} .
$$


Take $\mu \neq \lambda, \mu>0, \lambda>0$, rationals. Because of the independence of $S^{\mu}$ from $R$ and $X P\left\{S^{\mu}>R>M^{\mu}, S^{\lambda}>R>M^{\lambda}\right\}>0 ;$ also, $A \supset\left\{S^{\lambda}>R\right.$ $\left.>M^{\lambda}\right\}$ a.s. Therefore,

$$
P\left\{A, S^{\mu}>R>M^{\mu}\right\}=P\left\{A^{\mu}, S^{\mu}>R>M^{\mu}\right\}>0
$$

and by the argument just given, $A \supset\left\{S^{\mu}>R>M^{\mu}\right\}$ a.s. Hence if $P\left(A^{\lambda}\right)$ $>0$ for one $\lambda$ it is so for all other $\lambda$ and almost surely

proving (c).

$$
A \supset \underset{\lambda \text { rational }}{\bigcup}\left\{S^{\lambda}>R>M^{\lambda}\right\}=\{M<R\}
$$

REMARK. It is easy to construct examples of Hunt processes such that half the paths are continuous at the minimum and half jump out of the minimum. Thus the "pure" behavior of Lévy processes (and those processes that can be constructed therefrom by multiplicative and additive functionals) appears to be rather exceptional.

4. The strong Markov property at the minimum. It turns out that, for any real strong Markov process having a minimum the process $\{X(M+t), t>0\}$ is, given $(X(M), X(M-))$, a Markov process with transitions depending only on $X(M), X(M-)$. If, in addition, a zero-one law holds at $M$, this fact can be strengthened to assert that the "entrance law" of this process also depends only on $X(M), X(M-)$ and that the evolution of $\{X(M+t), t>0\}$ is conditionally independent of the pre- $M$ process, given $X(M), X(M-)$. This section contains the precise formulation and proof of these assertions as well as several examples.

Unless explicitly stated otherwise, $X=\left\{X_{t}, t \geqslant 0\right\}$ is a real right continuous strong Markov process $\left(\Omega, X_{t}, \theta_{t}, \mathscr{F}_{,} \mathscr{F}_{t}, P^{x}\right)$ with a lifetime $\zeta$ and transitions $P_{t}(x, d y)$ that map bounded Borel functions into Borel functions. Assumed, of course, is that $X$ is given an initial distribution $\mu$ and corresponding probability $P^{\mu}$ on the space of paths; the superscript $\mu$ will be omitted throughout. For simplicity assume also throughout that $M$, the time of the (last) minimum satisfies $P\{M<\zeta\}=1$. If $0<P\{M<\zeta\}<1$, one can obtain analogous results by conditioning on $\{M<\zeta\}$.

Define

$$
\begin{aligned}
M & =\sup \left\{t: X_{t} \leqslant X_{s}, \text { all } s \leqslant t\right\}, \\
M_{t} & =\sup \left\{r \leqslant t: X_{r} \leqslant X_{s}, \text { all } s \leqslant r\right\}, \\
I & =\inf _{s} X_{s}, \\
K_{t}(a ; x, f) & =E^{x}\left\{f\left(X_{t}\right), t<T_{a}\right\}, \quad T_{a}=\inf \left\{t>0: X_{t} \leqslant a\right\}, \\
H_{t}(a ; x, d y) & =K_{t}(a ; x, d y) h_{a}(y) / h_{a}(x), \quad h_{a}(x)=P^{x}\left\{T_{a}=\infty\right\} .
\end{aligned}
$$

Notice that 


$$
\begin{aligned}
E^{x}\left\{f\left(X_{t}\right), T_{a}=\infty\right\} & =E^{x}\left\{f\left(X_{t}\right), T_{a}>t, T_{a} \circ \theta_{t}=\infty\right\} \\
& =E^{x}\left\{f\left(X_{t}\right) h_{a}\left(X_{t}\right), T_{a}>t\right\} \\
& =K_{t}\left(a ; x, f h_{a}\right) .
\end{aligned}
$$

Assume $h_{a}(x)>0$ if $x>{ }^{\circ} a$. Let $M_{n}$, a discretization of $M$, be defined by

$$
\begin{aligned}
M_{n} & =k 2^{-n} \text { if }(k-1) 2^{-n}<M<k 2^{-n}, \\
& =0 \text { if } M=0 .
\end{aligned}
$$

The first lemma establishes the Markovian character of the post $M_{n}$ process, conditiona! on $I$.

LEMMA 4.1. Let $X=\left\{X_{t}, t \geq 0\right\}$ be a real standard Markov process. Then for bounded Borel $f$ and $t>s \geqslant 0$ :

$$
E\left\{f\left(X\left(M_{n}+t\right)\right) \mid \mathcal{F}\left(M_{n}+s\right)\right\}=H_{t-s}\left(I ; X\left(M_{n}+s\right), f\right) .
$$

Proof. The proof is a variant of the calculation of $[15, \mathrm{p} .304]$. Let

$$
\begin{gathered}
A \in \mathscr{F}\left(M_{n}+s\right), \quad B_{k}=\left\{k 2^{-n}<M<(k+1) 2^{-n}\right\}, \\
C(n, s)=\left\{M_{n}+s \leqslant(k+1) 2^{-n}+s\right\} \in \mathscr{F}\left(M_{n}+s\right) .
\end{gathered}
$$

By the definition of the sigma field $\mathscr{F}\left(M_{n}+s\right)$, there exists

$$
A^{\prime} \in \mathscr{F}\left((k+1) 2^{-n}+s\right)
$$

with

$$
A \cap C(n, s)=A^{\prime} \cap C(n, s)
$$

so $A \cap B_{n}=A^{\prime} \cap B_{n}$. But then

(4.4) $\int_{A} f\left(X\left(M_{n}+t\right)\right)=\int_{A\left(M_{n}=0\right\}} f\left(X_{t}\right)+\Sigma \int_{A B_{k}} f\left(X\left((k+1) 2^{-n}+t\right)\right)$

where

$$
\begin{aligned}
& \int_{A B_{k}} f\left(X\left((k+1) 2^{-n}+t\right)\right) d P \\
& =E\left\{f\left(X\left((k+1) 2^{-n}+t\right)\right) A^{\prime},\right. \\
& \left.\quad T_{Z(k)} \circ \theta_{k 2^{-n}}<2^{-n}, T_{Z(k+1)} \circ \theta_{(k+1) 2^{-n}}=\infty\right\} \\
& =E\left\{f\left((k+1) 2^{-n}+t\right) A^{\prime}, T_{Z(k)} \circ \theta_{k 2^{-n}}<2^{-n},\right. \\
& \left.\quad T_{Z(k+1)} \circ \theta_{(k+1) 2^{-n}}>s, T_{Z(k+1)} \circ \theta_{(k+1) 2^{-n+s}}=\infty\right\}
\end{aligned}
$$

with $Z(k)=\inf \left\{X_{s}: s<k 2^{-n}\right\}$. 
Condition the last expression in (4.5) on $\mathscr{F}\left((k+1) 2^{-n}+s\right)$ and use (4.2) to obtain

$$
\begin{aligned}
E\left\{A^{\prime}, T_{Z(k)} \circ \theta_{k 2^{-n}}\right. & <2^{-n}, T_{Z(k+1)} \circ \theta_{(k+1) 2^{-n}}>s \\
K_{t-s} & \left.\left(Z(k+1), X\left((k+1) 2^{-n}+s\right), f \circ h_{Z(k+1)}\right)\right\} .
\end{aligned}
$$

Multiply and divide $K_{t-s}$ in (4.6) by $h_{Z(k+1)}\left(X\left((k+1) 2^{-n}+s\right)\right.$ ) (which has been assumed positive) and simplify, to see that (4.6) is equal to

$$
\begin{gathered}
E\left\{A^{\prime}, T_{Z(k)} \circ \theta_{k 2-n}<2^{-n}, T_{Z(k+1)} \circ \theta_{(k+1) 2-n}>s, T_{Z(k+1) 2-n+s}=\infty,\right. \\
\left.H_{t-s}\left(Z(k+1), X\left((k+1) 2^{-n}+s\right), f\right)\right\} \\
=E\left\{A B_{k} H_{t-s}\left(I, X\left((k+1) 2^{-n}+s\right), f\right)\right\} .
\end{gathered}
$$

Sum on $k$ to complete the proof. The proof, of course, made use of the Markov property in the form [3, I.8.16].

Lemma 4.1 may be extended to the case where $s$ is a stopping time relative to the family $\left\{\mathscr{F}\left(M_{n}+s\right), s \geqslant 0\right\}$.

LEMMA 4.2. Let $X=\left\{X_{t}, t \geqslant 0\right\}$ be a real standard Markov process, and let $T$ be a stopping time relative to the family $\left\{\mathcal{G}_{s}, s \geqslant 0\right\}$, where $\mathcal{G}_{s}=\mathscr{F}\left(M_{n}+s\right)$. Then for bounded Borel $f$ and $t \geqslant 0$ :

$$
E\left\{f\left(X\left(M_{n}+T+t\right)\right) \mid \mathcal{G}_{T}\right\}=H_{t}\left(I ; X\left(M_{n}+T\right), f\right) .
$$

Proof. Once (4.7) and (4.8) below are established, the proof is essentially the same as that of Lemma 4.1: one conditions on $\mathscr{F}\left(T^{\prime}\right)$ instead of $\mathscr{F}\left((k+1) / 2^{n}+s\right)$ and uses the strong Markov property at $T^{\prime}$.

(4.7) If $T$ is a stopping time relative to $\mathcal{G}_{s}$, then for fixed $k$, there exists a stopping time $T$ for $\left\{\mathscr{F}_{s}, s \geqslant 0\right\}$ such that $(k+1) 2^{-n}+T=T^{\prime}$ on $\left\{M_{n}\right.$ $\left.=(k+1) 2^{-n}\right\}$.

(4.8) If $A \in \mathcal{G}_{T}$ and $k$ is fixed, let $T$ be the stopping time of (4.7). Then there exists a set $A^{\prime} \in \mathscr{F}\left(T^{\prime}\right)$ such that $A^{\prime} \cap\left\{M_{n}=(k+1) 2^{-n}\right\}=A \cap\left\{M_{n}\right.$ $\left.=(k+1) 2^{-n}\right\}$.

To prove (4.7) consider first the case of a stopping time $T$ having only a countable number of values $r_{1}<r_{2}<\cdots$. Then $T$ is a stopping time relative to $\left\{\mathscr{F}\left(M_{n}+r_{j}\right), j \geqslant 1\right\}$, whence $\left\{T=r_{j}\right\} \in \mathscr{F}\left(M_{n}+r_{j}\right)$. This last is equivalent to the assertion that, for each $k$,

$$
\left\{T=r_{j}\right\}\left\{M_{n}+r_{j}=r_{j}+(k+1) 2^{-n}\right\}=A_{j}\left\{M_{n}=(k+1) 2^{-n}\right\} \text { for each } j
$$

where $A_{j} \in \mathcal{F}\left((k+1) 2^{-n}+r_{j}\right)$. The sets $A_{1}, A_{2}, \ldots$ are disjoint on $\left\{M_{n}\right.$ $\left.=(k+1) 2^{-n}\right\}$, but possibly not on $\Omega$ so define $A_{1}^{\prime}=A_{1}, A_{2}^{\prime}=A_{2}-A_{1}, A_{3}^{\prime}$ $=A_{3}-\left(A_{1} \cup A_{2}\right)$ and so forth. Set $T^{\prime}=r_{j}+(k+1) 2^{-n}$ on $A_{j}^{\prime}$. Then $T^{\prime}$ is 
a stopping time relative to $\mathscr{F}\left((k+1) 2^{-n}+r_{j}\right)$ and so relative $t c$ s, and $T^{\prime}=T+(k+1) 2^{-n}$ on $\left\{M_{n}=(k+1) 2^{-n}\right\}$. If $T$ is a general $\left\{\mathscr{F}\left(M_{n}+s\right), s\right.$ $\geqslant 0\}$ stopping time, define $T_{m}=(j+1) 2^{-m}$ on $\left\{j 2^{-m}<T \leqslant(j+1) 2^{-m}\right\}$ so that $T_{m} \downarrow T$. For $k$ fixed, let $T_{m}^{\prime}$ be the $\mathscr{F}_{s}$ stopping time corresponding to $T_{m}$ as in the first part of the proof. Set $T^{\prime}=\lim \sup T_{m}$, again an $\mathscr{F}_{s}$ stopping time. Since $T_{m}=T_{m}^{\prime}$ on $\left\{M_{n}=(k+1) 2^{-n}\right\}, T=T^{\prime}$ on this set as well. Notice that the $T_{m}^{\prime}$ can be constructed so that $T_{m}^{\prime} \downarrow T^{\prime}$; for, $\left\{T_{m}^{\prime}\right\}$ does decrease on $\left\{M_{n}=(k+1) 2^{-n}\right\}$, and if it does not decrease on $\Omega$, replace by, $T_{1}^{\prime}$, $T_{1}^{\prime} \wedge T_{2}^{\prime}, \ldots$.

To check (4.8), assume first that $T$ is countable valued as in the proof of (4.7), and let $T^{\prime}$ be the stopping time constructed in (4.7) corresponding to $T$. Since $A \in \mathcal{G}_{T}, A \cap\left\{T=r_{j}\right\} \in \mathcal{F}\left(M_{n}+r_{j}\right)$ which means

$$
A \cap\left\{T=r_{j}\right\} \cap\left\{M_{n}+r_{j}=(k+1) 2^{-n}+r_{j}\right\}=\tilde{A}_{j}\left\{M_{n}=(k+1) 2^{-n}\right\}
$$

where

$$
\tilde{A}_{j} \in \mathscr{F}\left((k+1) 2^{-n}+r_{j}\right)=A_{j}^{\prime}\left\{T^{\prime}=r_{j}+(k+1) 2^{-n}\right\}\left\{M_{n}=(k+1) 2^{-n}\right\}
$$

where $A_{j}^{\prime} \in \mathscr{F}\left((k+1) 2^{-n}+r_{j}\right), A_{j}^{\prime} \subset\left\{T^{\prime}=r_{j}+(k+1) 2^{-n}\right\}$. The set $A^{\prime}$ $=\cup_{j} A_{j}^{\prime}$ is then in $\mathscr{F}\left(T^{\prime}\right)$. For general $T$, take $T_{m} \downarrow T$ as in the proof of (4.7) and let $T_{m}^{\prime}$ be the $\mathscr{F}_{s}$ stopping times of (4.7) corresponding to $T_{m}$, so chosen that $T_{m}^{\prime} \downarrow T^{\prime}$. If $A \in \mathcal{G}_{T}$, then $A \in \mathcal{S}\left(T_{m}\right)$ and so by what has just been proved, there exists $A_{j} \in \mathscr{F}\left(T_{j}^{\prime}\right)$ such that $A=A_{j}$ on $\left\{M_{n}=(k+1) 2^{-n}\right\}$. Set $A^{\prime}=\lim \sup A_{j} \in \mathscr{F}\left(T^{\prime}\right)$ to complete the proof.

LEMMA 4.3. The mappings $t \rightarrow H_{s}(I ; X(M+t), f)$ are right continuous on $(0, \infty)$ for each $s \geqslant 0$ and each continuous bounded $f$.

Proof. Since $M_{n} \downarrow M$, it suffices to show that $t \rightarrow H_{s}\left(I ; X\left(M_{n}+t\right), f\right)$ is right continuous. Moreover, since the process $Y_{t}=H_{s}\left(I ; M_{n}+t, f\right)$ is well measurable relative to the right continuous complete sigma fields

$$
\mathcal{G}_{s}=\mathscr{F}\left(M_{n}+s\right) \text {, }
$$

it is enough (see [12], [13]) to check that for all $\mathcal{G}_{t}$ stopping times $T_{m}, T$ with $T_{m} \downarrow T$ :

$$
\lim _{m \rightarrow \infty} E Y_{T_{m}}=E Y_{T} .
$$

But by Lemma 4.2,

$$
\begin{aligned}
\lim _{m \rightarrow \infty} E Y_{T_{m}} & =\lim _{m \rightarrow \infty} E\left\{f\left(X\left(M_{n}+T_{m}+s\right)\right)\right\} \\
& =E\left\{f\left(X\left(M_{n}+T+s\right)\right)\right\}=E Y_{T}
\end{aligned}
$$


The next proposition indicates that, given $I$, the post-minimum process is Markov with transitions $H_{t}(I ; x, d y)$. It should be compared with Theorem 5.1 of Meyer, Smythe and Walsh [15] for coterminal times.

Proposition 4.1. Let $\left\{X_{t}, t \geqslant 0\right\}$ be a real right continuous strong process. Then the post-minimum process $\{X(M+t), t>0\}$ satisfies

$$
E\{f(X(M+t)) \mid \mathscr{F}(M+s)\}=H_{t-s}(I ; X(M+s), f), \quad 0<s<t .
$$

Proof. A well-known fact, first discovered by Blackwell and Dubins [1], asserts that if $\left\{Y_{n}, n \geqslant 0\right\}$ is a bounded sequence of random variables converging a.s. to $Y$, and if $\left\{\mathscr{F}_{n}, n \geqslant 0\right\}$ is a decreasing family of sigma fields, then

$$
\lim _{n \rightarrow \infty} E\left\{Y_{n} \mid \mathscr{F}_{n}\right\}=E\left\{Y \mid \cap \mathscr{F}_{n}\right\} \text { a.s. }
$$

Since $\mathscr{F}\left(M_{n}+s\right) \downarrow \mathscr{F}(M+s)$, it is then immediate from (4.9) and Lemma 4.3 that

$$
\begin{aligned}
E\{f(X(M+t)) \mid \mathfrak{F}(M+s)\} & =\lim _{n \rightarrow \infty} E\left\{f\left(X\left(M_{n}+t\right)\right) \mid \mathscr{F}\left(M_{n}+s\right)\right\} \\
& =\lim _{n \rightarrow \infty} H_{t-s}\left(I ; X\left(M_{n}+s\right), f\right) \\
& =H_{t-s}(I ; X(M+s), f), \quad 0<s<t .
\end{aligned}
$$

If, for example, a zero-one law holds at $M$, then Proposition 4.1 can be strengthened.

Proposition 4.2. Let $\left\{X_{t}, t \geqslant 0\right\}$ be a real strong Markov process satisfying $P\{M<\zeta\}=1$ and

$$
\bigcap_{t>0} \sigma\{X(M+s), s \leqslant t\}=\sigma\{X(M), I\} \text { a.s. }(P)
$$

Let $Q_{s}(s, d y)$ be a regular conditional distribution of $P\{X(M+s) \in d y \mid(I$, $X(M))=x$ \}. If $0=s_{0}<s_{1}<\cdots<s_{k}$ and if $f$ is a bounded real Borel function of $k$ variables, then

$$
\begin{aligned}
& E\left\{f\left(X\left(M+s_{1}\right), \ldots, X\left(M+s_{k}\right)\right) \mid \mathscr{F}(M)\right\} \\
& =\int f\left(x_{1}, \ldots, x_{k}\right) H_{s_{k}-s_{k-1}}\left(I ; x_{k-1}, d x_{k}\right) \cdots H_{s_{2}-s_{1}}\left(I ; x_{1}, d x_{2}\right) \\
& H_{s_{2}-s_{1}}\left(I ; x_{1}, d x_{2}\right) Q_{s_{1}}\left((I, X(M)), d x_{1}\right) \text { a.s. }
\end{aligned}
$$

REMARK. $P\{M<\zeta\}=1$ can be replaced by $P\{M<\zeta\}>0$ at the price of further conditioning. The result should be compared to that of Pittenger and 
Shih [19] for coterminal times; here the post- $M$ proces ' ts transition functions that depend on $\omega$.

Proof. By Proposition 4.1 and a martingale convergence theorem,

$$
\begin{aligned}
E\{f(X(M+t)) \mid \mathscr{F}(M)\} & =\lim _{s \rightarrow 0} E\{f(X(M+t)) \mid \mathscr{F}(M+s)\} \\
& =\lim _{s \rightarrow 0} H_{t-s}(I ; X(M+s), f) .
\end{aligned}
$$

By (4.10), this last limit belongs to $\sigma\{X(M), I\}$ (modulo $P$ null sets), implying that

$$
E\{f(X(M+t)) \mid \mathscr{F}(M)\}=E\{f(X(M+t)) \mid X(M), I\} \text { a.s. }
$$

This being so, it follows for example that

$$
\begin{aligned}
E & \{g(X(M+s)) f(X(M+t)) \mid \mathscr{F}(M)\} \\
& =E\{g(X(M+s)) E\{f(X(M+t)) \mid \mathscr{F}(M+s)\} \mid \mathscr{F}(M)\} \\
& =E\left\{g(X(M+s)) H_{t-s}(I ; X(M+s), f) \mid \mathscr{F}(M)\right\} \\
& =\int g(x) f(y) H_{t-s}(I ; x, d y) Q((I, X(M)), d x) .
\end{aligned}
$$

Extension in the usual manner completes the proof.

The next three propositions provide examples of processes satisfying the hypotheses of Proposition 4.2.

Proposition 4.3. Let $\left\{X_{t}, t \geqslant 0\right\}$ be a real strong Markov process with right continuous paths and left limits such that $P\{M<\zeta\}=1$ and $P\{X(M)$ $\neq X(M-)\}=1$. Then (4.11) holds with $Q_{s}((I, X(M)), f)=H_{s}(I ; X(M), f)$.

ReMarK. Of course, if $I=X(M)$ (see $\$ 3$ for examples of this), then there is no dependence on $X(M-)$. In general, however, the dependence on $X(M-)$ cannot be omitted.

Proof. Let $J_{1}, J_{2}, \ldots$ be an enumeration of the jumps of $\left\{X_{t}\right\}$, so $M$ occurs at one of the $J_{i}$. If $A \in \cap_{s>0} \sigma\left\{X\left(J_{n}+t\right), t \leqslant s\right\}$, then $A=A^{\prime} \circ \theta_{J_{n}}$ for some $A^{\prime} \in \mathscr{F}_{0}$, so by the right continuity of the fields and the strong Markov property

$$
I_{A}=E\left\{I_{A} \mid \mathcal{F}\left(J_{n}\right)\right\}=P^{X\left(J_{n}\right)}\left\{A^{\prime}\right\} .
$$

Hence, if $A \in \cap_{s>0} \sigma\{X(M+t), t \leqslant s\}$, then $A=f\left(X\left(J_{n}\right)\right)$ on $\left\{M=J_{n}\right\}$ so (4.10) holds.

It remains only to check that $Q$ has the stated form. If

$$
Z(t)=\min \{X(t), X(t-)\},
$$

then 


$$
\begin{aligned}
& E f(X(M+s)) g(X(M), X(M-)) \\
& \quad=\sum E f\left(X\left(J_{n}+s\right)\right) g\left(X\left(J_{n}\right), \dot{X}\left(J_{n}-\right)\right) I\left\{M=J_{n}\right\} .
\end{aligned}
$$

The $n$th term in this sum is

$$
\begin{aligned}
& E\left\{f\left(X\left(J_{n}+s\right)\right) g\left(X\left(J_{n}\right), X\left(J_{n}-\right)\right) ; X_{u} \geqslant Z\left(J_{n}\right), u<J_{n} ;\right. \\
& \left.\quad X_{u}>Z\left(J_{n}\right), J_{n}<u<J_{n}+s ; \quad X_{u} \circ \theta_{J_{n}+s}>Z\left(J_{n}\right), u>0\right\} .
\end{aligned}
$$

Apply the strong Markov property at the time $J_{n}+s$ and reduce the resulting expression as in the proof of Lemma 4.1 to obtain as an expression for the $n$th term

$$
E\left\{g\left(X\left(J_{n}\right), X\left(J_{n}-\right)\right) I\left\{M=J_{n}\right\} H_{s}\left(I ; X\left(J_{n}\right), f\right)\right\} .
$$

Sum on $n$ to complete the proof.

In preparation for the next proposition, define a killed Lévy process to be any strong Markov process $\left\{\gamma_{t}, t \geqslant 0\right\}$ for which there exists a Lévy process $\left\{X_{t}\right\}$ and a random time $R$ such that

$$
\begin{aligned}
\gamma_{t} & =X_{t}, & t<R, \\
& =\Delta, & t \geqslant R .
\end{aligned}
$$

Here we include the possibility that $R$ depends on quantities independent of $\left\{X_{t}\right\}$. Killed Lévy processes include, for example, all processes that are transformations of some Lévy process via a multiplicative functional (see [3, Chapter III]). There is the evident version of the next proposition for processes that are time changes of a Lévy process; we spare the reader the details.

Proposimion 4.4. Let $X=\left\{X_{t}, t \geqslant 0\right\}$ be a killed Lévy process, $P\{M<\zeta\}$ $=1$. Then (4.11) holds.

Proof. According to the results of $\$ 3$, the sigma field

$$
\bigcap_{s>0} \sigma\{X(M+t)-X(M), t \leqslant s\}
$$

is trivial, and this implies (4.10).

The final proposition contains results of Jacobsen [11] and Williams [22], who treated regular diffusions by other methods.

Proposimon 4.5. Let $X=\left\{X_{t}, t \geqslant 0\right\}$ be a standard real Markov process, $P\{M<\zeta\}=1$, satisfying

$$
P\{X(t) \leqslant X(t-), \text { all } t<\zeta\}=1 .
$$

Then (4.11) holds. 
Proof. By the results of Blackwell and Dubins mentioned in the proof of Proposition 4.1, and by Proposition 4.1

$$
\begin{aligned}
E\{f(X(M+t)) \mid \mathscr{F}(M)\} & =\lim _{s \downarrow 0} E\{f(X(M+t+s)) \mid \mathscr{F}(M+s)\} \\
& =\lim _{s \downarrow 0} H_{t}(I ; X(M+s), f) .
\end{aligned}
$$

However, by (4.13), $X$ upon leaving its minimum passes through every point in an interval $[X(M), X(M)+\varepsilon]$ provided $\varepsilon$ is small (how small depends on $\omega)$. Since $\lim _{s \downarrow 0} H_{t}(I ; X(M+s), f)$ exists, $\lim _{x \downarrow X(M), x>X(M)} H_{t}(I ; x, f)$ exists so the limit in question can depend only on $I, X(M)$. The proof can now be completed in exactly the same way as Proposition 4.2. Alternatively it is not difficult to deduce (4.10) from the observations just made.

One concluding remark: if $X$ should leave its minimum in several "ways" (e.g., some paths jump out, others leave continuously), and if, conditional on the manner of leaving, a zero-one law holds then a variant of Proposition 4.2 holds, conditional on the manner of leaving.

5. Zero-one laws and last exit times. The main results of this section give the precise criteria for a Lévy process to admit zero-one laws at the last leaving of an interval. Connections between the post-minimum process and the process after last leaving an interval were discussed in $\$ 1$. As in the case of the minimum, the analysis is closely connected with the study of how the paths behave at the random time of interest. Some of the techniques of 83 will therefore the relevant in the present situation; indeed, use of the minima of various processes turns out to be a convenient tool.

Another ingredient of the solution is the concept isolated in [16]: a real Lévy process admits continuous passages upward if

$$
P\left\{X\left(T_{x}\right)=X\left(T_{x}-\right)\right\}>0 \text { for some } x>0
$$

where

$$
T_{x}=\inf \left\{t>0: X_{t}>x\right\} .
$$

This definition is interesting, of course, only when the process hits points; i.e.,

$$
P\left\{H_{x}<\infty\right\}>0 \text { for some (hence all [9]) } x
$$

where

$$
H_{x}=\inf \left\{t>0: X_{t}=x\right\} .
$$

The criteria for a process to hit points are due to Kesten [9]; see also [4]. It was shown in [16] that if (5.1) holds for one $x>0$, then it holds for all $x>0$. The notion of continuous passages downward has the obvious formulation. 
According to [16], the only processes with continuous passages both upward and downward are those with Gaussian components. If a process (which hits points) admits continuous passages in neither direction, then it is easy to see that such a process jumps upward and downward over a point infinitely often just before hitting it (see [16, Theorem 1.1]). Define

$$
\begin{aligned}
& A_{n}^{+}=A_{n}^{+}(x)=\left\{\omega: X\left(H_{x}-s\right)>x, \text { some } s<\frac{1}{n}\right\}, \\
& A_{n}^{-}=A_{n}^{-}(x)=\left\{\omega: X\left(H_{x}-s\right)<x, \text { some } s<\frac{1}{n}\right\} .
\end{aligned}
$$

If $X$ has neither continuous passages upwards or downwards, then

$$
P\left(\bigcap_{n} A_{n}^{+}\right)=P\left(\bigcap_{n} A_{n}^{-}\right)=P\left\{H_{x}<\infty\right\} .
$$

On the other hand, if $X$ has a Gaussian component, $\cap_{n} A_{n}^{+}$and $\cap_{n} A_{n}^{-}$are disjoint with

$$
P\left(\bigcap_{n} A_{n}^{+}\right)+P\left(\bigcap_{n} A_{n}^{-}\right)=P\left\{H_{x}<\infty\right\} .
$$

Finally, if $X$ has continuous passages upward but not downward, then

$$
\bigcap_{n} A_{n}^{+} \text {is empty and } P\left\{\bigcap_{n} A_{n}^{-}\right\}=P\left\{H_{x}<\infty\right\} \text {. }
$$

With these facts at hand, we can now describe the sample function behavior of a process as it last leaves the interval $(-\infty, a]$. Let

$$
\begin{aligned}
L_{a, t} & =\sup \left\{s<t: X_{s} \leqslant a\right\}, \\
& =0 \text { if the set above is empty. }
\end{aligned}
$$

Proposition 5.1. Let $X=\left\{X_{t}, t \geqslant 0\right\}$ be a real Lévy process such that neither $X$ nor $-X$ is a subordinator. Then

$$
P\left\{X\left(L_{a, t}\right)=X\left(L_{a, t}-\right), 0<L_{a, t}<t\right\}>0
$$

if and only if $X$ admits continuous passages upward.

ReMarK. The hypothesis that neither $X$ nor $-X$ be a subordinator guarantees $P\left\{0<L_{a, t}<t\right\}>0$ for all $a, t$. The last exit of a subordinator from $(-\infty, a]$ corresponds to its first entrance into $[a, \infty)$ and the latter phenomenon being well understood (see $[9, \S 6]$ or [6]), the analogue of Proposition 5.1 for these cases is easy and uninteresting. 
The proof of the proposition is based on the principle that a process leaves a set for the last time "in the same way" that the dual process hits that set for the first time.

Proof. If $X$ does not hit points, then $P\left\{X_{t}\right.$ or $X_{t}$ - equals $a$ for some $t>0\}=0$; therefore in this case $P\left\{X\left(L_{a, t}\right)=X\left(L_{a, t}-\right)=a, 0<L_{a, t}<t\right\}$ $=0$ and the issue is settled. So, suppose that $X$ hits points. Let $X^{\lambda}$ be the process derived from $X$ by exponential killing at $S^{\lambda}$ as in Proposition 3.1. If $U^{\lambda}(x, A)=\int e^{-\lambda t} P_{t}(x, A) d t$ is the usual $\lambda$-potential measure, then since $X$ hits points, $U^{\lambda}(x, \cdot)$ has a density $u^{\lambda}(x, y)=u^{\lambda}(y-x)$ which is continuous and strictly positive on $(-\infty, \infty)$ (Bretagnolle [4]). Let $Y=\left\{Y_{t}, t>0\right\}$ be $X^{\lambda}$ reversed from its lifetime $S^{\lambda}$ :

$$
Y(t)=X^{\lambda}\left(S^{\lambda}-t\right), \quad 0<t<S^{\lambda} .
$$

According to the theory of Nagasawa [18] (see also [14]), $Y$ has transition functions

$$
P_{t}^{\lambda}(x, d y) u^{\lambda}(0, y) / u^{\lambda}(0, x)
$$

where $P_{t}^{\lambda}(x, d y)=e^{-\lambda t} P_{t}(x, d y)$ are the transition functions of the process $P^{\lambda}$ dual to $X^{\lambda}$. Set $h(y)=u^{\lambda}(0, y)$, so $Y$ is a Doob $h$-path transform of $X^{\lambda}$. According to a basic relation relating the $h$-transform of a process to the original process due to Doob [5] (see also [10])

$$
\hat{E}_{h}^{\lambda, x}\{A, t<\zeta\}=E^{\lambda, x} I_{A} h\left(X_{t}\right) / h\left(X_{0}\right)
$$

where $E^{\lambda, x}$ denotes expectation relative to the usual measures in general Markov theory corresponding to the transitions $f_{t}^{\lambda}(x, d y), E_{h}^{\lambda, x}$ is expectation corresponding to the transitions (5.8), the $X_{t}^{\prime}$ 's are the coordinate maps in the usual function space representation and $A$ is a set in $\mathscr{F}_{f}^{0}$. Choose $A$ $=\left\{\omega: X\left(T_{a}\right)=X\left(T_{a}-\right), T_{a}<t\right\}$ where $T_{a}=\inf \left\{t>0: X_{t} \in(-\infty, a]\right\}$. By results of [16] summarized in the beginning of this section, if $X$ has no continuous downward passages (a.s. relative to $\left\{P^{x}\right\}$ ), then for each $t$ $\hat{E}^{\lambda, x} I_{A} h\left(X_{t}\right) / h\left(X_{0}\right)=0, x>a$. Therefore by (5.9) if $\hat{T}_{a}=\inf \left\{s>0: Y_{s}\right.$ $\in(-\infty, a]\}$

$$
P^{0}\left\{Y\left(\hat{T}_{a}\right)=Y\left(\hat{T}_{a}-\right), 0<\hat{T}_{a}<S^{\lambda}\right\}=0 .
$$

But if $L_{a}^{\lambda}=\sup \left\{t: X_{t}^{\lambda} \leqslant a\right\}$, then $Y\left(\hat{f}_{a}-\right)=X^{\lambda}\left(L_{a}^{\lambda}\right)$ and $Y\left(\hat{T}_{a}\right)=X^{\lambda}\left(L_{a}^{\lambda}-\right)$ so

$$
P^{0}\left\{X^{\lambda}\left(L_{a}^{\lambda}\right)=X^{\lambda}\left(L_{a}^{\lambda}-\right), 0<L_{a}^{\lambda}<S^{\lambda}\right\}=0 .
$$

That is, if $L_{a}^{\lambda}$ is the time of the last exit from $(-\infty, a]$ before $S^{\lambda}$, then $P\left\{X\left(L_{a}^{\lambda}\right)=X\left(L_{a}^{\lambda}-\right), 0<L_{a}^{\lambda}<S^{\lambda}\right\}=0$. A localization argument similar to 
that of Theorem 3.1 effects the replacement of the $S^{\lambda}$ by the fixed times $t$, settling the issue under the assumption that $X$ has no continuous upward passages. If, finally, $X$ does have continuous upwards passages an entirely similar argument, which, however, also uses the fact that $h$ is strictly positive everywhere, again leads to the desired conclusion.

Proposition 5.1 opens the way to a zero-one law at the last leaving of a halfline. The notation is that of Proposition 5.1 and, as before, discussion of the subordinator case will be omitted because of its relative triviality.

Proposition 5.2. Let $X=\left\{X_{t}, t \geqslant 0\right\}$ be a real process with stationary independent increments such that neither $X$ nor $-X$ is a subordinator. Suppose that either

(5.10) $X$ does not admit continuous passages upward or

(5.11) $X$ has no upward jumps.

Let $\mathfrak{L}_{s}=\sigma\left\{X\left(L_{a, t}+u\right)-X\left(L_{a, t}\right), u \leqslant s\right\}, \mathfrak{L}_{0+}=\cap_{s>0} \mathfrak{L}_{s}$. If $A \in \mathfrak{L}_{0+}$, then $P\left\{A \mid 0<L_{a, t}<t\right\}=0$ or 1 . If (5.10) and (5.11) fail, then the zero-one property fails.

REMARK. In case (5.11) or (5.10) fails, an argument similar to the one in the coming proof shows that if $A \in \mathfrak{L}_{0+}$, then

$$
P\left\{A \mid 0<L_{a, t}<t, X\left(L_{a, t}\right)=X\left(L_{a, t}-\right)\right\}=0 \text { or } 1
$$

and

$$
P\left\{A \mid 0<L_{a, t}<t, X\left(L_{a, t}\right) \neq X\left(L_{a, t}-\right)\right\}=0 \text { or } 1 .
$$

The sets on which the conditioning is made both have positive probability in this case.

Proof. If (5.10) holds, then on $\left\{0<L_{a, t}<t\right\}$ the process always jumps out of $(-\infty, a]$ at $L_{a, t}$. Hence in this case the zero-one law follows from the strong Markov property at the jump times and the Blumenthal zero-one law. For the case where there are no upward jumps, the process satisfies

$$
X\left(L_{a, t}\right)=X\left(L_{a, t}-\right)=a
$$

on the set $\left\{0<L_{a, t}<t\right\}$. Let $X^{\lambda}$ be the usual exponentially killed process, and $L_{a}^{\lambda}$ the last time if any that it is below $a$. Let $M_{\varepsilon}$ be the time at which $\left\{X^{\lambda}(s), s>L_{a}^{\lambda}+\varepsilon\right\}$ attains its minimum. An analysis similar to that of Lemma 3.1 shows that $\left\{X^{\lambda}\left(M_{\varepsilon}+t\right)-X\left(M_{\varepsilon}\right), t>0\right\}$ is independent of $\mathscr{F}\left(M_{\varepsilon}-\right)$. By Proposition 5.1, $X$ leaves $(-\infty, a]$ for the last time continuously, so as $\varepsilon \rightarrow 0, X^{\lambda}\left(M_{\varepsilon}+t\right) \rightarrow X^{\lambda}\left(L_{a}^{\lambda}+t\right)$ on $\left\{0<L_{a}^{\lambda}<S^{\lambda}\right\}$. An argument like that of Lemma 3.2(b) shows that if $A \in \cap_{t>0} \sigma\left\{X^{\lambda}\left(L_{a}^{\lambda}+s\right)-X\left(L_{a}^{\lambda}\right), s<t\right\}$, then $P\left\{A \mid 0<L_{a}^{\lambda}<S^{\lambda}\right\}=0$ or 1 . The argument of Theorem 3.1 replaces the $S^{\lambda}$ 
by fixed times, completing the proof under the assumption that either (5.10) or (5.11) holds. Finally, suppose both (5.10) and (5.11) fail. Then the dial process has continuous passages downward and has downward jumps as well. Proposition 2.1 of [16] shows that the dual process (killed at $S^{\lambda}$ ) on first passing below level $a$ will hit that level with a positive probability and will also jump over that level with positive probability. A time riversal like that of Proposition 5.1 shows that as a consequence $0<P\left\{X^{\lambda}\left(L_{a}^{\lambda}\right)=X\left(L_{a}^{\lambda}-\right), 0\right.$ $\left.<L_{a}^{\lambda}<S^{\lambda}\right\}<P\left\{0<L_{a}^{\lambda}<S^{\lambda}\right\}$, and it is easy to complete the proof of the converse from here.

\section{REFERENCES}

1. D. Blackwell and L. Dubins, Merging opinions with increasing information, Ann. Math. Statist. 33 (1962), 882-886. MR 26 \#062.

2. R. M. Blumenthal, An extended Markov property, Trans. Amer. Math. Soc. 85 (1957), 52-72. MR 19, 468.

3. R. M. Blumenthal and R. K. Getoor, Markov processes and potential theory, Academic Press, New York, 1968. MR 41 \# 9348.

4. J. Bretagnolle, Résultats de Kesten sur les processus da accroisements indépendantes (Séminaire de Probabilités. V, Univ. de Strasbourg), Lecture Notes in Math., vol. 191, Springer-Verlag, Berlin and New York, 1971, pp. 21-36.

5. J. L. Doob, Conditional Brownian motion and the boundary limits of harmonic functions, Bull. Soc. Math. France 85 (1957), 431-458. MR 22 \#844.

6. B. Fristedt, Sample functions of stochastic processes with stationary, independent increments, Advances in Probability III, Dekker, New York, pp. 241-396.

7. I. I. Gikhman and A. V. Skorokhod, Introduction to the theory of random processes, "Nauka", Moscow, 1965; English transl., Saunders, Philadelphia, Pa., 1969. MR 33 \#6689; 40 \# 923.

8. G. A. Hunt, Some theorems concerning Brownian motion, Trans. Amer. Math. Soc. 81 (1956), 294-319. MR 18, 77.

9. H. Kesten, Hitting probabilities of single points for processes with stationary independent increments, Mem. Amer. Math. Soc. No. 93 (1969). MR 42 \#6940.

10. H. Kunita and T. Watanabe, Notes on transformations of Markov processes connected with multiplicative functionals, Mem. Fac. Sci. Kyushu Univ. Ser. A 17 (1963), 181-191. MR 29 \#661.

11. M. Jacobsen, Splitting times for Markov processes and a generalized Markov property for diffusions, Z. Wahrscheinlichkeitstheorie 30 (1974), 27-43.

12. J.-F. Mertens, Sur la théorie des processus stochastiques, C. R. Acad. Sci. Paris Ser. A-B 268 (1969), A495-A496. MR 39 \#4922.

13. P.-A. Meyer, Le retournement du temps d'après Chung et Walsh (Séminaire de Probabilités. III, Univ. de Strasbourg), Lecture Notes in Math., vol. 88, Springer-Verlag, Berlin and New York, 1969, p. 143.

14. - Processus de Markov: La frontière de Martin, Lecture Notes in Math., vol. 77, Springer-Verlag, Berlin and New York, 1968. MR 39 \#7669.

15. P. A. Meyer, R. T. Smythe and J. B. Walsh, Birth and death of Markov processes, Proc.

Sixth Berkeley Sympos. Math. Statist. and Probability, Vol. III, 1972, pp. 293-305.

16. P. W. Millar, Exit properties of stochastic processes with stationary independent increments, Trans. Amer. Math. Soc. 178 (1973), 459-479. MR 47 \#9731.

17. - Sample functions at a last exit time, Z. Wahrscheinlichkeitstheorie 34 (1976), 91-111.

18. M. Nagasawa, Time reversions of Markov processes, Nagoya Math. J. 24 (1964), 177-204. MR 29 \#6542. 
19. A. O. Pittenger and C. T. Shih, Coterminal families and the strong Markov property, Trans. Amer. Math. Soc. 182 (1973), 1-42. MR 49 \#1600.

20. B. A. Rogozin, The local behavior of processes with independent increments, Teor. Verojatnost. i Primenen. 13 (1968), 507-512 = Theor. Probability Appl. 13 (1968), 482-486. MR 39 \#3593.

21. J. B. Walsh, Markov processes and their functionals in duality, Z. Wahrscheinlichkeitstheorie und Verw. Gebiete 24 (1972), 229-246. MR 48 \#398.

22. D. Williams, Path decomposition and continuity of local time for one-dimensional diffusions, Proc. London Math. Soc. 28 (1974), 738-768.

Departmient of Statistics, University of California, Berkeley, California 94720 